Original Article

\title{
Femoral cam deformity due to anterior capsular force: A theoretical model with MRI and cadaveric correlation
}

\author{
Cara Beth Lee ${ }^{\mathrm{a}, *}$, Hillard T. Spencer ${ }^{\mathrm{b}}$, Kirsten F. Nygaard ${ }^{\mathrm{a}}$ \\ ${ }^{a}$ Center for Hip Preservation, Virginia Mason Medical Center, 925 Seneca Street, Lindeman Pavilion, Level 6, Seattle, WA 98111, USA \\ ${ }^{\mathrm{b}}$ Southern California Permanente Medical Group, Orthopedics Department, 3460 E, La Palma Avenue, Anaheim, CA 92806, USA
}

\section{A R T I C L E I N F O}

\section{Article history:}

Received 8 May 2016

Accepted 20 June 2016

Available online 5 July 2016

\section{Keywords:}

Femoroacetabular impingement

FAI

Cam deformity

Hip impingement

Hip capsule

\begin{abstract}
A B S T R A C T
Background: Cam deformity is associated with epiphyseal extension onto the anterosuperior femoral head-neck before physeal closure. A century ago, anatomists speculated that this femoral prominence acts as a pulley bar to withstand capsular compression in hip extension with pressure concentrated where the zona orbicularis (ZO) joins the iliofemoral ligament (IFL). An animal model has shown that growth plates deflect laterally and distally when exposed to forces perpendicular to growth. These observations raise the question of whether capsular pressure against the epiphysis can stimulate cam formation.

Purpose: The purposes are to measure: (1) the distance from the ZO/IFL confluence to the maximal epiphyseal extension (MEE) and cam apex; and (2) acetabular depth at this location, since less coverage increases capsular contact on the physis.

Methods: MRI scans of 39 subjects (47 hips) were measured. Acetabular depth was compared between those with and without a cam deformity. Secondarily, anatomic findings were correlated on a cadaveric specimen.

Results: The cam apex and MEE were adjacent to the ZO/IFL confluence in all subjects (mean, $6.3 \mathrm{~mm}$ ). Controlling for sex, acetabular depth was less $(12.5 \%, p=0.012)$ in the group with cam deformity. Contact points were confirmed in the specimen.

Conclusions: The cam apex and MEE occur at the ZO/IFL confluence in the thickest region of the anterosuperior capsule and vary with acetabular depth. This supports a theoretical model postulating that capsular forces against an immature epiphysis may induce cam formation, particularly in individuals who repetitively tension the anterior capsule.
\end{abstract}

(C) 2016 Prof. PK Surendran Memorial Education Foundation. Published by Elsevier, a division of Reed Elsevier India, Pvt. Ltd. All rights reserved.

\section{Introduction}

Femoroacetabular impingement (FAI) is increasingly recognized as a cause of hip pain and risk factor for osteoarthritis. ${ }^{1}$ The femoral cam lesion is a developmental deformity ${ }^{2-5}$ that is implicated in the labrochondral damage in FAI. ${ }^{1,6}$ It is associated with abnormal extension of the epiphysis onto the anterosuperior proximal femur. ${ }^{5}$ This epiphyseal extension precedes formation of the cam lesion before physeal closure. ${ }^{3}$ Although cam morphology is more prevalent in athletes, the mechanism for its development is unknown.

\footnotetext{
Abbreviations: FAI, femoroacetabular impingement; ZO, zona orbicularis; IFL, iliofemoral ligament; MEE, maximal epiphyseal extension; EER, epiphyseal extension ration; ARA, acetabular roof angle.

* Corresponding author. Tel.: +1 206223 7530; fax: +1 2065155846 .

E-mail address: Carabeth.lee@virginiamason.org (C.B. Lee).
}

Anatomists first characterized a bony prominence at the anterior femoral neck in 1899 , calling it the 'eminentia articularis colli femoris', or 'eminentia'. ${ }^{7-9}$ It was speculated that this fibrocartilage-covered eminentia functions as a pulley bar for the anterior hip capsule when the joint is in a position of extension. ${ }^{9}$ Specifically, the most compressive force occurs where the zona orbicularis ( $\mathrm{ZO}$ ) blends with the iliofemoral ligament (IFL) at the medial side (head-neck junction) of the eminentia. ${ }^{9}$ Bone morphology is influenced by local and systemic mechanical loading and modulated by the direction, degree and timing of the forces at the epiphyseal growth plates. ${ }^{10-12}$ Forces perpendicular to the direction of growth have been shown in both an animal model $^{11}$ and a computer simulation ${ }^{13}$ to result in lateral displacement of the epiphysis and periosteal apposition.

Noting that the cam lesion is most pronounced at the anterosuperior femoral head-neck junction, 5,14 particularly between 1 and 2 o' clock, which correlates to the thickest part of the 
anterior capsule, ${ }^{15}$ raises the question whether anterior capsular pressure in this location may stimulate epiphyseal extension and cam formation. As a first step to explore this question, this study investigates the location where the fibers of the iliofemoral ligament and zona orbicularis coalesce with respect to: (1) the maximal epiphyseal extension in the proximal femur in subjects with and without a femoral cam lesion (alpha angle less than $55^{\circ}$ ), and (2) the apex of the cam deformity in those with the lesion. We then compared acetabular measures between the two groups, since acetabular depth influences the trajectory and degree of contact the capsule has against the proximal femur. The null hypotheses are that there is no correlation between the location of the capsular fiber confluence and the cam or epiphyseal extension, and there is no difference in acetabular depth between groups.

\section{Methods}

After institutional review board approval, we reviewed the database of new patients evaluated for hip pain at a single institution over a 2-year period from October 2012 through September 2014. We selected those who had undergone MRI scans at our facility and who were between the ages of 15 and 25 to ensure physeal closure and adequate visualization of the physeal scar, and to minimize the possibility of secondary arthritic change. Subjects were excluded if they had previous surgery, trauma, systemic illness that would affect hip growth, or inadequate image sets.

During the study time period, 1424 new patients were evaluated for hip pain, 185 between the ages of 15 and 25. Forty-four underwent MRI in our institution; five were excluded based on the criteria described above. This left 39 subjects (47 hips) for evaluation. Thirty-six hips had alpha angle equal to or greater than 55 (cam group) and 11 had alpha angles less than 55 (normal offset group) measured on MRI radial views.

Our MRI protocol is unique in that patients lie supine with their hips in a position of comfort, rather than forcing internal rotation to correct for femoral anteversion. As such, the location of the hip capsule and proximal femur are considered to be in their natural, resting position. The study was performed on an 18-channel $1.5 \mathrm{~T}$ MR system (Magnetom Symphony, Siemens, Erlangen, Germany) using a small flex coil to wrap the hip and large body matrix coil for pelvis imaging. Two-dimensional proton density fat-saturated turbo-spin echo coronal $(\mathrm{TR} / \mathrm{TE}=3000 / 37 \mathrm{~ms}$, matrix size $=320 \times$ 320 , slice thickness $=3 \mathrm{~mm}, \mathrm{FOV}=180)$ and true sagittal $(\mathrm{TR} /$ $\mathrm{TE}=3000 / 28 \mathrm{~ms}$, matrix size $=320 \times 320$, slice thickness $=3 \mathrm{~mm}$, FOV $=180)$ views were obtained, as well as a 3-dimensional space sequence $(\mathrm{TR} / \mathrm{TE}=1200 / 18 \mathrm{~ms}$, matrix size $=250 \times 256$, slice thickness $=0.80 \mathrm{~mm}, \mathrm{FOV}=200$ ). Radial and axial oblique images were generated from multiplanar reconstructions correcting for the rotation of the femur. Eighteen radial views were produced with the first slice at the $12 \mathrm{o}$ ' clock position of the coronal plane and rotating clockwise in $10^{\circ}$ intervals around, and perpendicular to, the femoral neck axis. Axial oblique reconstructions were obtained parallel to the femoral neck at $3 \mathrm{~mm}$ slice thickness. Using an Intellispace PACS worksite (Phillips; Andover, MA), the alpha angle and epiphyseal extension were measured on all 10 radial views that comprise the anterosuperior quadrant of the proximal femur (12-3 o' clock) where the epiphyseal extension and cam lesion are most pronounced. ${ }^{4,5}$ The radial slice and values for the maximal epiphyseal extension and apex of the cam lesion, if present, were recorded. To correct for variation in femoral head diameter, epiphyseal extension is expressed as a ratio of the distance from the medial femoral head to the epiphyseal line divided by the femoral head diameter as described by Siebenrock and colleagues (Fig. 1a)..$^{5}$ The acetabular roof coverage was measured on radial views and determined by the angle between a line extending through the center of the femoral head and neck to the acetabulum (a continuation of the line used for the alpha angle measurement) and a second line from the center of the femoral head tangential to the most anterior point of the bony rim (Fig. 1b).

The MRI DICOM files were imported into Osirix (Pixmeo; Geneva, Switzerland), which numerically displays 3-dimensional locations in the scan. The 0 point on the $x, y, z$-axis corresponds to the isocenter of the magnet when the scan is obtained. This point may vary between subjects, but the 3-dimensional coordinates are accurate for within subject/scan measures. The 3-dimensional coordinates of the maximal epiphyseal extension and the cam apex were recorded from the radial view (Fig. 1c), and the confluence of the superior band of the iliofemoral ligament with the zona orbicularis on the axial oblique views (Fig. 1d). ${ }^{16}$ To minimize bias, the two points were recorded at different sessions.

Hips were classified according to the presence or absence of a cam deformity, which was defined as an alpha angle of $55^{\circ}$ or greater on any radial slice. The distance measured on MRI between the ZO/IFL and the point of maximum epiphyseal extension was compared between groups with a $t$-test. This calculation was repeated for the distance between the ZO/IFL and the apex of the cam deformity in the subset of patients with alpha angle of $55^{\circ}$ or greater. Finally, the acetabular coverage angle at the point of maximum epiphyseal extension was compared between groups using univariable and multivariable regression. The reliability of the MRI measurement technique was evaluated by repeating blinded measurements on ten hips at an interval of 3 months after the original measurement by the same examiner. Ten subjects were randomly selected for repeated measures of the distance on MRI between the ZO/IFL and the point of maximum epiphyseal extension. These measures were then compared to the original measures for these subjects to judge the reliability of the measurement technique. Two methods of assessing reliability were performed - first, direct comparison of the mean distance measurements between measurement episodes, and second, the 95\% limits of agreement method of Bland and Altman, and then comparing mean differences in measurements, as well as calculating the Bland-Altman limits of agreement between measurements. Statistical significance was defined as $p<0.05$; all tests were two-sided.

In the second part of the study, a cadaveric specimen from a 50 year-old male with a cam lesion and no radiographic arthritis was imaged with CT, fluoroscopy and MRI, and then dissected to correlate the imaging findings with the anatomic structures. Finally, the capsule was examined histologically to define the fiber orientation at the confluence of the zona orbicularis and the superior band of the iliofemoral ligament.

\section{Results}

There was a higher proportion of males in the cam group compared to the normal offset group but no difference between the groups with respect to age, body mass index, side of involvement, or epiphyseal extension ratio (Table 1 ). In all subjects, the distance from the confluence of the superior band of the iliofemoral ligament and zona orbicularis was in close proximity to the maximal epiphyseal extension $(5.7 \mathrm{~mm}$ in the cam group and $8.1 \mathrm{~mm}$ in the normal offset group; mean $6.3 \mathrm{~mm}$ ). There was no statistically significant difference in this distance between groups $(p=0.27)$. Similarly, in subjects with an alpha angle greater than $55^{\circ}$, the capsular confluence closely corresponded to the location of the apex of the cam deformity (mean, $4.98 \mathrm{~mm}$; 95\% CI 4.00-5.96).

At the location of maximal epiphyseal extension, the acetabular roof angle was larger in the group with normal offset compared to the cam group ( $70.9^{\circ}$ vs. $64.1^{\circ}$, mean difference $\left.6.8^{\circ}, p=0.04\right)$. In multivariable analysis, controlling for sex, the acetabular depth 

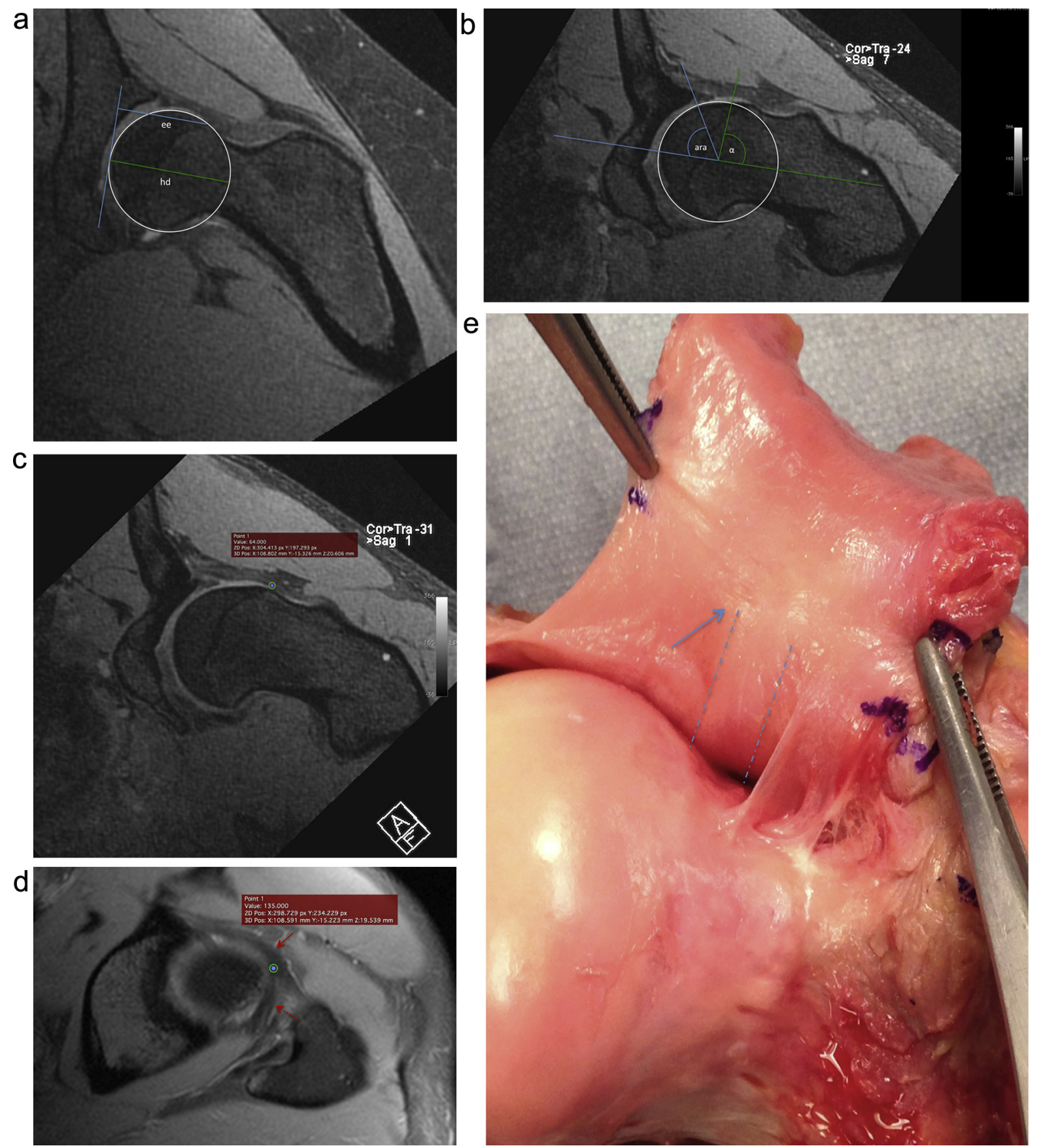

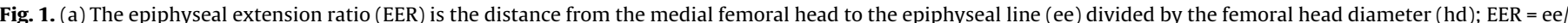

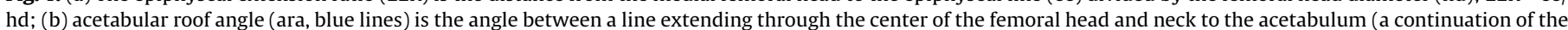

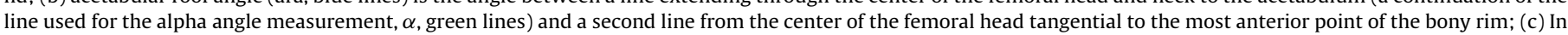

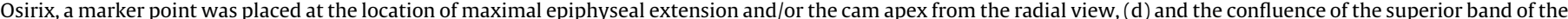

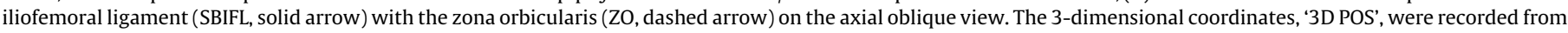

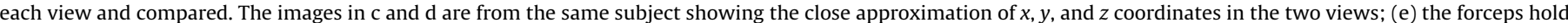

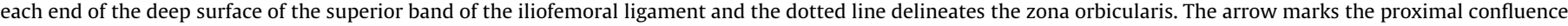
point of the ZO and SBIFL correlating to the location measured on MRI (marker point in figure d).

was significantly less $(12.5 \%, p=0.012)$ in the cam group compared to the normal offset group. For reliability testing of the measurements, there was no significant difference between the original measures and the repeated measures of the distance between ZO/IFL and the point of maximum epiphyseal extension (mean difference $0.1 \mathrm{~mm}, p=0.804$ ). The $95 \%$ limits of agreement by Bland-Altman method were -3.472 to 3.708 with no difference in variance by Pitman's test, $p=0.14$.

In the cadaveric specimen, the superior band of the iliofemoral ligament (SBIFL) was a distinct thickening that became taut in extension and external rotation (Fig. 2a), as well as external rotation with flexion. With dynamic examination under fluoroscopy, the superior band of the iliofemoral ligament closely overlay the maximal epiphyseal extension and apex of the cam lesion on a simulated $45^{\circ}$ Dunn view. The capsule was released along the acetabular origin and anterior intertrochanteric insertion and folded superolaterally to examine the zona orbicularis (Figs. 1e and $2 b)$. In neutral position, the confluence point of the $\mathrm{ZO}$ with the deep fibers of the SBIFL corresponded with the location of the cam lesion on the femur (Fig. 2b). The femur was then sectioned 
Table 1

Subject demographics.

\begin{tabular}{llll}
\hline & Alpha angle $>55$ & Alpha angle $<55$ & $p$-Value \\
\hline Gender (\% female/male) & $29 / 71$ & $91 / 9$ & $0.0008^{*}$ \\
Age (average in years) & 20.1 & 18.8 & 0.24 \\
Body mass index & 23.67 & 21.8 & 0.12 \\
EER & 0.82 & 0.79 & 0.07 \\
ARA at max EER & 64.1 & 70.9 & $0.04^{*}$ \\
Side (\% right/left) & $58 / 42$ & $45 / 55$ & 0.51 \\
Alpha angle average & 67.2 & & \\
ARA at max alpha angle & 62.9 & & \\
\hline
\end{tabular}

EER, epiphyseal extension ratio; ARA, acetabular roof angle.

Statistical significance at $p<0.05$

through this marker point in a plane simulating an MRI radial view. The marker coincided with the location of epiphyseal extension (Fig. 2c). Histological examination at the capsular confluence demonstrated the perpendicularly interlaced fibers of the zona orbicularis and iliofemoral ligament (Fig. 2d).

\section{Discussion}

The MRI findings in this study confirm that the points of maximal epiphyseal extension and cam deformity on the femoral head-neck junction occur adjacent to the ZO/IFL confluence in the anterosuperior capsule. Acetabular depth is lower in subjects with a cam lesion, which exposes the epiphysis to greater capsular contact than subjects with deeper coverage. Gross and histologic examination of a cadaveric specimen correlate the anatomic landmarks with the MRI locations on the proximal femur and capsule.

Bone morphology is influenced by mechanical loading, and epiphyseal growth plates, in particular, are susceptible to the direction, amount and timing of the pressures to which they are exposed. ${ }^{10-12}$ In a rabbit model, shear pressure perpendicular to the direction of growth, i.e., parallel to the growth plate itself, led to deflection of the cartilage columns of the growth plate and lateral displacement of the epiphysis in a manner that resembles the "slow slipping of an epiphysis" on radiographs. ${ }^{11}$ Siebenrock and colleagues reviewed MRI scans of 15 subjects with symptomatic cam FAI and found that lateral epiphyseal extension on the anterosuperior quadrant of the femoral head-neck was associated with decreased head-neck offset compared to matched controls. ${ }^{5}$ Subsequent work demonstrated that cam morphology is preceded by epiphyseal extension ${ }^{3}$ in association with sports activity during

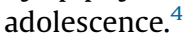

In 1931, Odgers ${ }^{9}$ expounded on previous anatomists' observations of the proximal femoral 'eminentia', 7,8 a consistent prominence on the anterosuperior femoral neck that is recognizable as a variant of a femoral cam lesion. ${ }^{1}$ He speculated that the eminentia
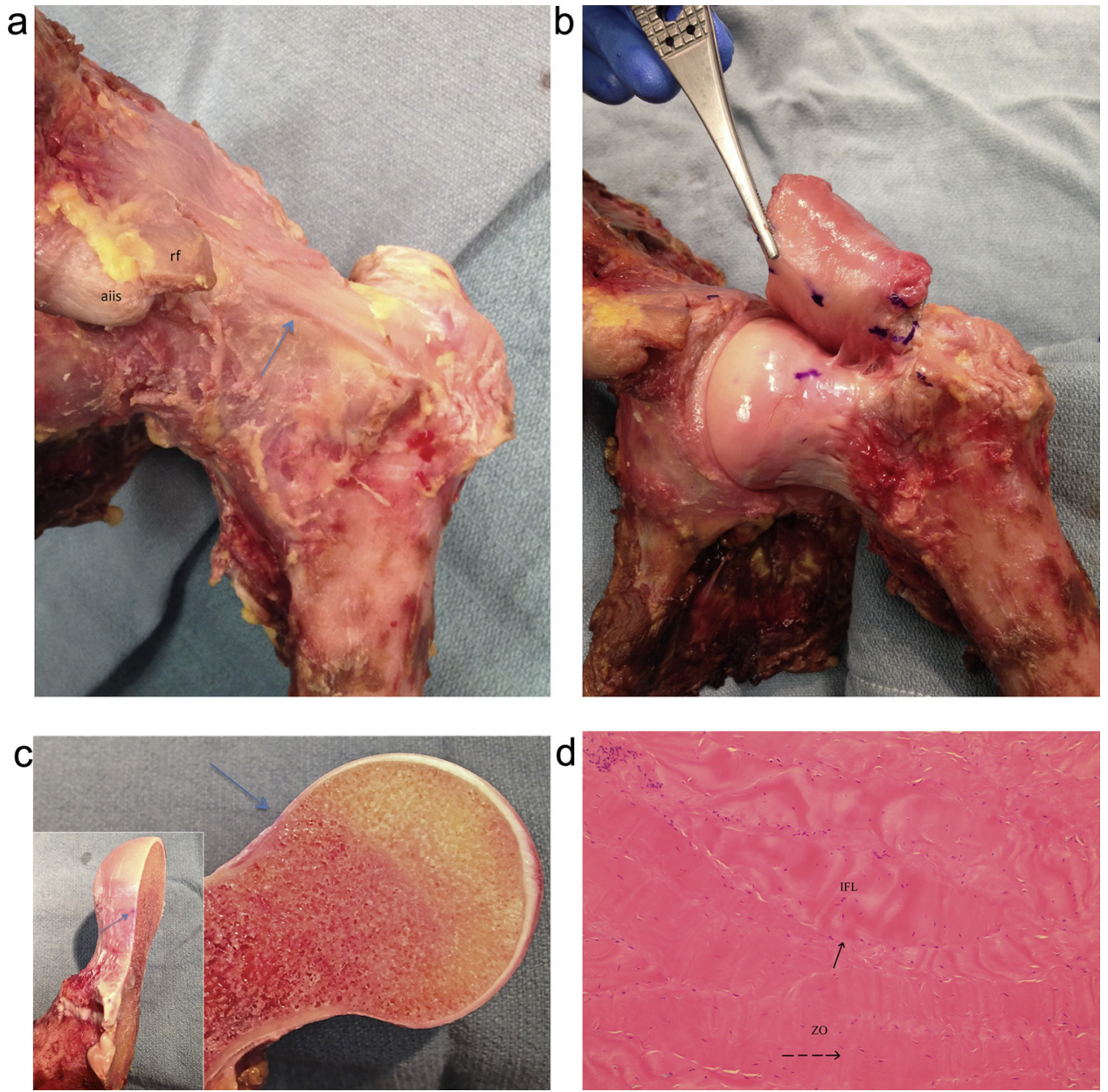

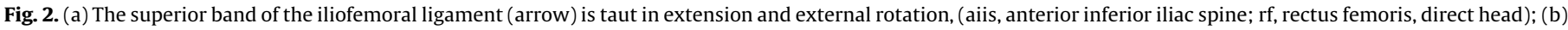

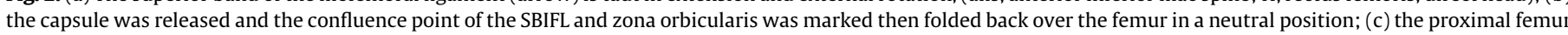

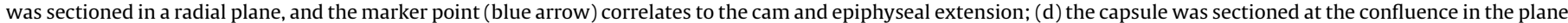

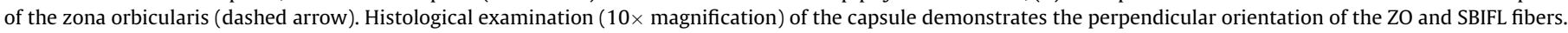
The solid arrow shows the demarcation between $\mathrm{ZO}$ and SBIFL fibers. 
acts as a 'pulley bar' for the anterior capsule with the greatest friction and pressure occurring at the junction of the vertical limb of the iliofemoral ligament with the encircling fibers of the zona orbicularis. The zona orbicularis is a circular or horseshoe-shaped region of the capsule that runs perpendicular to the femoral neck axis. $^{16,17}$ It is located at the boundary of sphericity of the femoral head $^{18}$ and functions to resist distraction of the hip. ${ }^{19}$ The iliofemoral ligament is composed of two bands ${ }^{17}$; the inferior band limits hip extension, ${ }^{16,20}$ while the superior band is a checkrein for external rotation in flexion and extension, even when combined with adduction and abduction. ${ }^{16,17}$ It is the strongest and stiffest of the hip capsular ligaments. ${ }^{21}$ The IFL acts as a tension spring to maintain stable equilibrium and conserve energy in standing with the hip in full extension ${ }^{22}$ and counters the anterior and caudally-directed force of the femoral head during weightbearing. ${ }^{23}$ The decrease in femoral anteversion after walking age in childhood is attributed to this posteriorly directed iliofemoral ligament force. $^{24}$

In a study demonstrating that the thickest portion of the iliofemoral ligament is between the 1 and 2 o' clock positions of the anterosuperior femoral neck, ${ }^{15}$ which corresponds to the most common location of the femoral cam lesion, ${ }^{4,14}$ the authors suggest that the ligament becomes thick in this location in response to the cam lesion. We propose the opposite - the cam prominence develops in response to capsular pressure on the growth plate. Cam lesions have a higher prevalence in athletes, ${ }^{4}$ particularly those who participate in soccer, ice hockey, American football and basketball. ${ }^{2,3,25,26}$ These sports frequently involve positions that tension the iliofemoral ligament in external rotation, such as the skating stride in hockey, passing, receiving, and shooting in soccer, and the defensive stance in football and basketball. When taut, the IFL exerts pressure against the anterior head-neck junction and physis, and the degree of contact will vary with acetabular depth. A more spherical femoral head-neck junction and increased offset have been observed in deeper sockets compared to dysplastic hips, ${ }^{27}$ and decreased offset is a common finding in patients with dysplasia. ${ }^{28}$ Although cam articular damage occurs as a result of impingement when the hip is in a position of flexion and internal rotation, in this theory, the cam lesion itself forms as a result of repeated hip extension and external rotation during development.

There are several limitations to this investigation. The first is the small sample size that was drawn from subjects with symptomatic hips. The second limitation is that the normal head-neck offset group was predominantly female. However, after controlling for gender, the main findings were supported. Since this was a preliminary exploration of the theory, we confined the study to available MRI scans obtained with the unique protocol at our facility and opted to restrict the age range to minimize the occurrence of secondary changes to the hip. Expansion of this project will require a wider range of subjects and controls. The biggest limitation of this study is that it is a substantial conceptual leap to propose that demonstrating close proximity of these structures implies causality between them. Despite these factors, the focal location of the cam lesion on the anterosuperior femoral neck argues for a local loading stimulus. A computer simulation of bone morphogenesis suggests that far-field, whole bone loads determine bone cross-sectional size, but cross-sectional shape is influenced by local periosteal surface load with perpendicular forces stimulating growth. ${ }^{13}$ To explore this theory further, it will be necessary to determine the amount of force required to alter physeal development, the effects of intermittent vs. constant force, the force generated by the anterior capsule in different hip positions and whether this produces tensile, shear forces across the epiphysis or direct, compressive pressure at the surface of the growth plate. An added challenge is the lack of an animal model to serve as a comparator to the obligate bipedal gait and lumbopelvic alignment of humans.

The limits of this study notwithstanding, the theory of repetitive capsular force against the immature growth plate as a stimulus for cam formation is a compelling explanatory model, because it unifies multiple, diverse observations that have been made about the cam deformity, such as:

1) The consistent location of the cam lesion on the anterosuperior head-neck junction. ${ }^{4,6,14}$

2) More anterior location of cam lesion with increased femoral version. ${ }^{14}$

3) Increased femoral head-neck offset in protrusio acetabuli or acetabular overcoverage where the femoral neck is protected from capsular contact. ${ }^{27}$

4) Conversely, the association of decreased femoral head-neck offset with acetabular dysplasia. ${ }^{28}$

5 ) The high prevalence of cam lesions in sports, ${ }^{25}$ particularly those that involve external rotation. ${ }^{2,26}$

6) Less deformity in those who started sports later in adolescence. ${ }^{2}$

7) No recurrence of deformity after osteoplasty in skeletally mature patients. ${ }^{29}$

8) Absence of cam prominence in anthropoids who ambulate with bent-hip bent-knee gait, and thus do not tension the anterior capsule. .,30 $^{9}$

\section{Conclusion}

The MRI and cadaveric findings in this study confirm that the apex of cam deformity and maximal epiphyseal extension occur adjacent to the thickest region of the anterior capsule where the superior band of the iliofemoral ligament coalesces with the zona orbicularis. The perpendicularly oriented fibers form a tether point that tensions the capsule against the femoral head-neck junction as the hip externally rotates. Compared to subjects with normal headneck offset, those with cam lesions had less acetabular coverage, and thus increased capsular contact at the point of maximal deformity. These findings lend preliminary support to a theoretical model postulating that capsular forces against an immature growth plate may contribute to cam formation, particularly in individuals who participate in activities that tension the anterior capsule. The theory requires further validation with biomechanical and developmental models. If substantiated, it may have implications for activity recommendations during growth.

\section{Funding}

This research did not receive any specific grant from funding agencies in the public, commercial, or not-for-profit sectors.

\section{Conflicts of interest}

The authors have none to declare.

\section{Acknowledgments}

The authors would like to thank Michael Cecil and Zachary Pizano with the imaging components of the study and Dr. Steven Ruhoy for assistance with the cadaveric and histologic evaluation.

\section{Appendix A. Supplementary data}

Supplementary data associated with this article can be found, in the online version, at doi:10.1016/j.jor.2016.06.012. 


\section{References}

1. Ganz R, Parvizi J, Beck M, Leunig M, Notzli H, Siebenrock KA. Femoroacetabular impingement: a cause for osteoarthritis of the hip. Clin Orthop Relat Res. 2003;417:112-120.

2. Agricola R, Heijboer MP, Ginai AZ, et al. A cam deformity is gradually acquired during skeletal maturation in adolescent and young male soccer players: a prospective study with minimum 2-year follow-up. Am J Sports Med. 2014;42: 798-806.

3. Siebenrock KA, Behning A, Mamisch TC, Schwab JM. Growth plate alteration precedes cam-type deformity in elite basketball players. Clin Orthop Relat Res. 2013;471:1084-1091.

4. Siebenrock KA, Ferner F, Noble PC, Santore RF, Werlen S, Mamisch TC. The cam-type deformity of the proximal femur arises in childhood in response to vigorous sporting activity. Clin Orthop Relat Res. 2011;469:3229-3240.

5. Siebenrock KA, Wahab KH, Werlen S, Kalhor M, Leunig M, Ganz R. Abnormal extension of the femoral head epiphysis as a cause of cam impingement. Clin Orthop Relat Res. 2004;418:54-60.

6. Tannast M, Goricki D, Beck M, Murphy SB, Siebenrock KA. Hip damage occurs at the zone of femoroacetabular impingement. Clin Orthop Relat Res. 2008:466:273-280.

7. Sudeck P. Zur Anatomie and Aetiologie der Coxa vara adolescentium. Arch Klin Chirurg. 1899;Bd. LIX:504-524.

8. Fick R. Handbuch der Anatomie u. Mechanik der Gelenke. 1904.

9. Odgers PN. Two details about the neck of the femur. (1) The Eminentia. (2) The Empreinte. J Anat. 1931;65:352-362.

10. Frost HM. Biomechanical control of knee alignment: some insights from a new paradigm. Clin Orthop Relat Res. 1997;335:335-342.

11. Arkin AM, Katz JF. The effects of pressure on epiphyseal growth; the mechanism of plasticity of growing bone. J Bone Jt Surg Am. 1956;38:1056-1076.

12. Rauch F. Bone growth in length and width: the Yin and Yang of bone stability. J Musculoskelet Neuronal Interact. 2005;5:194-201.

13. Carpenter RD, Carter DR. The mechanobiological effects of periosteal surface loads. Biomech Model Mechanobiol. 2008;7:227-242.

14. Ross JR, Bedi A, Stone RM, et al. Intraoperative fluoroscopic imaging to treat cam deformities: correlation with 3-dimensional computed tomography. Am J Sports Med. 2014;42:1370-1376.

15. Weidner J, Buchler L, Beck M. Hip capsule dimensions in patients with femoroacetabular impingement: a pilot study. Clin Orthop Relat Res. 2012;470: 3306-3312
16. Wagner FV, Negrao JR, Campos J, et al. Capsular ligaments of the hip: anatomic histologic, and positional study in cadaveric specimens with MR arthrography. Radiology. 2012;263:189-198.

17. Martin HD, Savage A, Braly BA, Palmer IJ, Beall DP, Kelly B. The function of the hip capsular ligaments: a quantitative report. Arthroscopy. 2008;24:188-195.

18. Malagelada F, Tayar R, Barke S, Stafford G, Field RE. Anatomy of the zona orbicularis of the hip: a magnetic resonance study. Surg Radiol Anat. 2015;37:11-18.

19. Ito H, Song Y, Lindsey DP, Safran MR, Giori NJ. The proximal hip joint capsule and the zona orbicularis contribute to hip joint stability in distraction. J Orthop Res. 2009;27:989-995.

20. Hidaka E, Aoki M, Muraki T, Izumi T, Fujii M, Miyamoto S. Evaluation of stretching position by measurement of strain on the ilio-femoral ligaments: an in vitro simulation using trans-lumbar cadaver specimens. Man Ther. 2009;14:427-432.

21. Hewitt JD, Glisson RR, Guilak F, Vail TP. The mechanical properties of the human hip capsule ligaments. J Arthroplasty. 2002;17:82-89.

22. Aspden RM, Rudman KE, Meakin JR. A mechanism for balancing the human body on the hips. J Biomech. 2006;39:1757-1759.

23. Kolodny A. The architecture and the blood supply of the head and neck of the femur and their importance in the pathology of fractures of the neck. J Bone Jt Surg Am. 1925;7:575-597.

24. Somerville EW. Persistent foetal alignment of the hip. J Bone Jt Surg Br. 1957;39: 106-113.

25. Nepple JJ, Vigdorchik JM, Clohisy JC. What is the association between sports participation and the development of proximal femoral cam deformity? A systematic review and meta-analysis. Am J Sports Med. 2015. [epub ahead of print]

26. Nepple JJ, Brophy RH, Matava MJ, Wright RW, Clohisy JC. Radiographic findings of femoroacetabular impingement in National Football League combine athletes undergoing radiographs for previous hip or groin pain. Arthroscopy. 2012;28: 1396-1403.

27. Steppacher SD, Tannast M, Werlen S, Siebenrock KA. Femoral morphology differs between deficient and excessive acetabular coverage. Clin Orthop Relat Res. 2008;466:782-790.

28. Clohisy JC, Nunley RM, Carlisle JC, Schoenecker PL. Incidence and characteristics of femoral deformities in the dysplastic hip. Clin Orthop Relat Res. 2009;467:128-134.

29. Gupta A, Redmond JM, Stake CE, Finch NA, Dunne KF, Domb BG. Does the femora cam lesion regrow after osteoplasty for femoroacetabular impingement? Two-year follow-up. Am J Sports Med. 2014;42:2149-2155.

30. Ng VY, Ellis TJ. More than just a bump: cam-type femoroacetabular impingement and the evolution of the femoral neck. Hip Int. 2011;21:1-8. 